\title{
Metodología b-learning aplicada en la enseñanza de ultrasonido clínico
}

\author{
Nicolas Rojas-Muñoz ${ }^{1}$, Hans Clausdorff-Fiedler ${ }^{2}$, Felipe Riquelme-Morales ${ }^{2}$, Joaquín Vidal-Castillo ${ }^{3 *}$ y \\ Carlos Basaure-Verdejo ${ }^{2}$
}

${ }^{1}$ Departamento de Medicina de Urgencia, Facultad de Medicina, Universidad de la Frontera, Temuco; ${ }^{2}$ Departamento de Medicina de Urgencia, Facultad de Medicina, Pontificia Universidad Católica de Chile, Santiago; ${ }^{3}$ Facultad de Medicina, Universidad Católica de la Santísima Concepción, Concepción. Chile

\section{Resumen}

Introducción: La Trombosis venosa profunda (TVP) en extremidades inferiores (EI) es una consulta frecuente en los servicios de urgencia y el uso de ultrasonido clínico (USC) es fundamental para su detección. Objetivo: Evaluar el método b-learning para entrenar a Médicos de Urgencia (MU) y residentes en el diagnóstico de TVP proximal de El por medio de USC en pacientes que consulten por sospecha de TVP proximal. Métodos: Estudio prospectivo de corte transversal. $27 \mathrm{MU}$ y residentes recibieron la capacitación. Se reclutó a 101 pacientes que consultaron al servicio de urgencia con sospecha de TVP en El. Se excluyeron a aquellos con antecedentes de TVP, usuarios de tratamiento anticoagulante e imposibilidad de ultrasonido doppler realizado por el médico radiólogo considerado el estándar de oro. Resultados: 17 (17\%) pacientes con TVP proximal. 8 (8\%) pacientes con TVP distal. 3 pacientes indeterminados, por lo que fueron excluidos.Después del entrenamiento, MU sin experiencia, lograron una sensibilidad del 100\% (IC 95\% 80\% -100\%) y una especificidad del 93,75\% (IC 95\% 86\% -98\%). Conclusión: Se logra una buena correlación en comparación con el estándar de oro. Con capacitación mínima cualquier MU puede identificar y descartar fácilmente una TVP proximal de El.

Palabras clave: Educación médica. Ultrasonido. Trombosis venosa. Medicina de urgencias.

\section{Successful concise b-learning methodology to teach compression ultrasound technique}

\begin{abstract}
Introduction: Deep vein thrombosis (DVT) in the lower extremities (LE) is a frequent consultation in the emergency services and the use of clinical ultrasound (USC) is essential for its detection. Objective: To evaluate the b-learning method to train Emergency Physicians (EM) and residents in the diagnosis of proximal DVT of LE by means of USC in patients who consult for suspected proximal DVT. Methods: Prospective cross-sectional study. 27 MU and residents received the training. 101 patients who consulted the emergency department with suspected DVT in LE were recruited. Those with a history of DVT, users of anticoagulant treatment and the impossibility of a Doppler ultrasound performed by the radiologist considered the gold standard were excluded. Results: 17 (17\%) patients with proximal DVT. 8 (8\%)
\end{abstract}

\section{Correspondencia:}

*Joaquín I. Vidal Castillo

E-mail: joaquin.vc15@gmail.com
Fecha de recepción: 26/03/21

Fecha de aceptación: 09/07/21

DOI: 10.24875/REIE.21000027
Disponible en internet: 13-09-2021

Rev Educ Investig Emer. 2021;3(3):133-139 www.medicinadeemergencias.com

作 bajo la licencia CC BY-NC-ND (http://creativecommons.org/licenses/by-nc-nd/4.0/). 
patients with distal DVT. 3 indeterminate patients, so they were excluded. After training, inexperienced MUs achieved a sensitivity of $100 \%(95 \% \mathrm{Cl} 80 \%-100 \%)$ and a specificity of $93.75 \%$ (95\% Cl 86\% -98\%). Conclusion: A good correlation is achieved compared to the gold standard. With minimal training, any MU can easily identify and rule out proximal LE DVT.

Key words: Education medical. Ultrasonography. Venous thrombosis. Medicine. Emergency.

\section{Introducción}

El ultrasonido clínico (USC) se ha convertido en una herramienta útil, rápidamente disponible y con gran utilidad en la práctica diaria de la medicina de urgencia (MU). Tanta relevancia a cobrado que el Colegio Estadounidense de Graduados en Educación Médica (ACGME) desde 2012 lo reconoce como herramienta fundamental, requiriendo capacitación en su uso durante la residencia ${ }^{1}$. En 2009, el Colegio Estadounidense de Médicos de Emergencia (ACEP) publicó una declaración con respecto a 11 aplicaciones principales del USC en la práctica diaria en los departamentos de urgencia ${ }^{2}$. Siendo una de las principales la detección de trombosis venosa profunda (TVP) en extremidades inferiores. Aunque los objetivos de su uso son bastante claros, el proceso de capacitación y aprendizaje no está bien unificado.

Descartar la TVP es un problema frecuente en los departamentos de urgencia. No realizar un diagnóstico temprano conlleva un riesgo de morbilidad y mortalidad, sobre todo en relación con la generación de embolias pulmonares, donde una de las principales etiologías son las TVP. El examen físico para su detección carece de precisión, pero su identificación por USC podría ser clave para el diagnóstico ${ }^{3}$. De hecho, de todos los pacientes con sospecha de TVP, solo el $20 \%$ tendrá diagnóstico confirmado 4 . Por lo tanto, a pesar de la mejora de las estrategias de diagnóstico, como las reglas de predicción clínica, el dímero $D$ y las técnicas avanzadas de imagen que incluyen angiotomografía computarizada y la flebografía, el diagnóstico de la TVP aún depende de la ecografía para su confirmación. En lugares donde la disponibilidad de imágenes es limitada, su aplicación de forma segura se convierte en un verdadero desafío para el médico de urgencia.

Hasta la fecha, la técnica de compresión es el examen de USC más frecuentemente descrito para la detección de TVP. Sin embargo, el método de capacitación, aprendizaje y práctica necesarios para realizar un estudio correcto no está bien descrito. El ACEP sugiere un curso completo de dos días y la finalización de
25-50 procedimientos revisados por pares para garantizar la calidad del examen².

Además, hay una amplia variedad de métodos descritos en la literatura desde la práctica aislada y mínima de solo 10 minutos hasta 6 horas de conferencia, más 24 horas de práctica adicional para completar la capacitación $^{5,6}$. En todos ellos resalta la interacción con el instructor, que permite el trabajo con grupos pequeños y contribuye a lograr un conocimiento consolidado de las habilidades en USC?.

El propósito de este estudio es evaluar un método b-learning para entrenar médicos de urgencia en el diagnóstico de TVP proximal por medio del uso de USC en pacientes que se presentan al departamento de urgencia con dolor en las piernas.

El método b-learning, definido como la combinación entre el aprendizaje presencial tradicional y el aprendizaje electrónico, ya sea de forma sincrónica o asincrónica, se presenta como un enfoque alternativo, demostrando efectividad y heterogeneidad en las fórmulas aplicadas $^{8,9}$, incluyendo el área de medicina de urgencias $^{10}$, siendo prometedor para la educación médica y permitiendo sintetizar ventajas de ambos estilos de aprendizaje ${ }^{8,11}$.

\section{Métodos}

\section{Diseño}

Se realizó un estudio transversal prospectivo en el servicio de urgencias de un centro médico universitario urbano con un promedio de 70.000 visitas al año. Un grupo de médicos y residentes de urgencia sin conocimientos de ultrasonido fueron entrenados para el diagnóstico de TVP proximal. Al finalizar el entrenamiento, se invitó a los pacientes adultos que se presentaban al servicio de urgencias con síntomas sugerentes de TVP de extremidades inferiores para participar en el estudio.

Los criterios de exclusión fueron:

- Pacientes con trombosis conocida al momento de la evaluación o en los últimos tres meses.

- Paciente en tratamiento anticoagulante durante más de 48 horas. 
- Imposibilidad de obtener una ecografía formal en el mismo día en el centro de estudio.

- Sospecha de trombosis de las extremidades superiores

- Pacientes con antecedentes crónicos de trombosis o portadores de catéter de diálisis en la pierna sintomática.

A todos aquellos pacientes que cumplieron los criterios de inclusión se les realizó USC de extremidades inferiores, por medio de una técnica ultrasonografía compresiva extendida para la detección de TVP proximal, luego se rellenó un formulario con sus resultados y por último se comparó contra la ecografía Doppler compresiva de extremidades inferiores realizada por el radiólogo, la que se considera estándar de oro.

\section{Protocolo de entrenamiento}

Se implementó un protocolo b-learning, en el cual 27 médicos, entre ellos especialistas en $\mathrm{MU}$, y residentes de segundo (PGY2) y tercer año (PGY3) de MU. Todos con nula experiencia en USC, fueron capacitados en la técnica de ultrasonografía compresiva extendida para determinar la presencia de TVP proximal ${ }^{12}$. El protocolo consistió en:

- Sesión online: clase videada asincrónica de $30 \mathrm{mi}-$ nutos realizada por un médico experto con estudios de posgrado en USC con la colaboración de un residente de $\mathrm{MU}$, que cubrió la revisión del equipo requerido, indicaciones, técnica, anatomía normal con y sin ultrasonido, análisis de casos clínicos y errores frecuentes en la interpretación de imágenes. Esta sesión se realizó mediante una clase videada asincrónica, cuyo enlace se entregó a cada participante.

- Sesión práctica: se realizó un día después de la sesión teórica y consistió en un ejercicio práctico de 90 min dirigido por un médico con estudios de posgrado en USC, donde se utilizó una máquina de ultrasonido con un paciente simulado sano. Se abordó la configuración de la máquina, transductor que utilizar, posicionamiento óptimo del paciente, reconocimiento de referencias anatómicas, interpretación de imagen de ultrasonido, errores de interpretación y finalizó con un cuestionario audiovisual con casos clínicos reales.

Finalizada esta capacitación los médicos se consideraron aptos para enrolar pacientes y realizar el examen clínico.

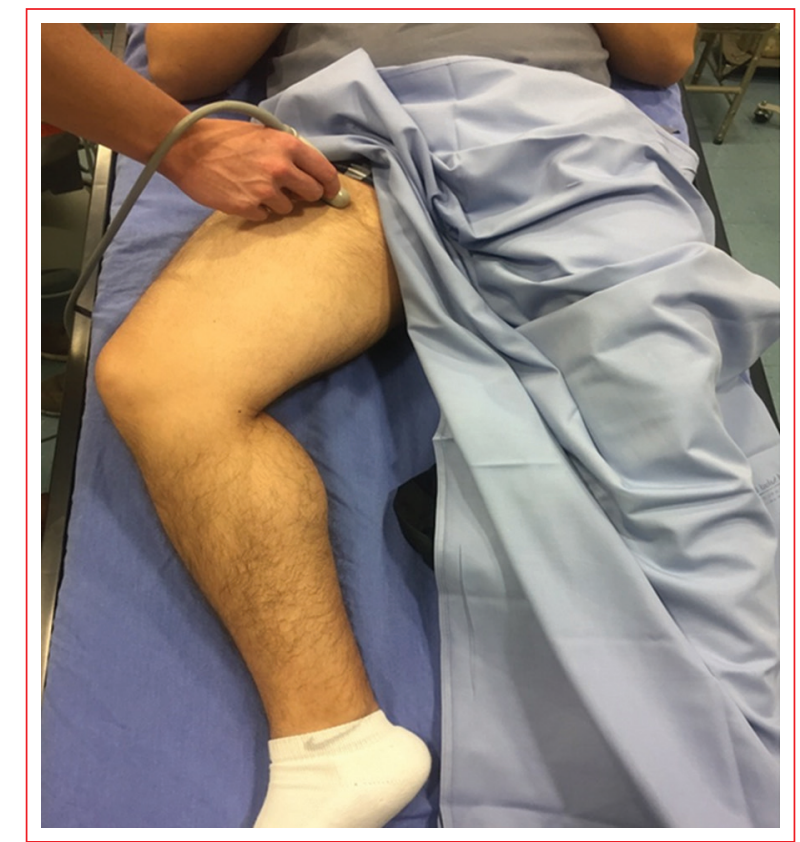

Figura 1. Evaluación de la unión de la vena safena mayor con la vena femoral común.

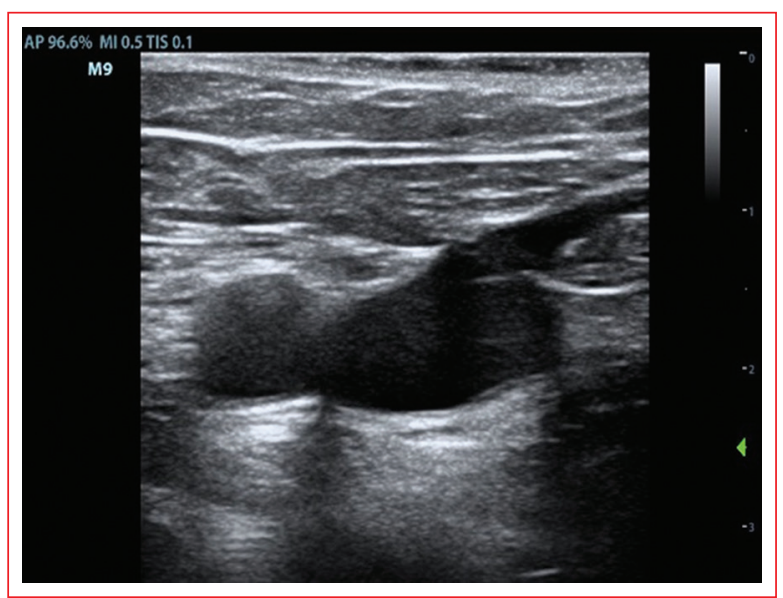

Figura 2. Imagen ecográfica vena femoral común no colapsada.

\section{Adquisición de la imagen e interpretación}

Una vez que el paciente firma el consentimiento informado, dado que el médico tratante sospechó TVP, se contactó a un médico capacitado para realizar la exploración ecográfica. Cabe destacar que ellos nunca compartieron información del paciente. Este último fue ciego al cuadro clínico, incluido síntomas y examen físico. 


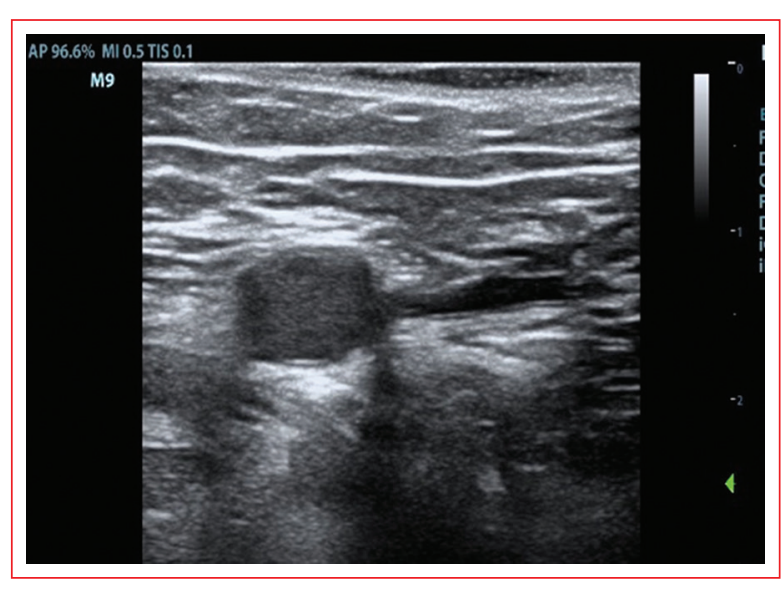

Figura 3. Imagen ecográfica vena femoral común colapsada.

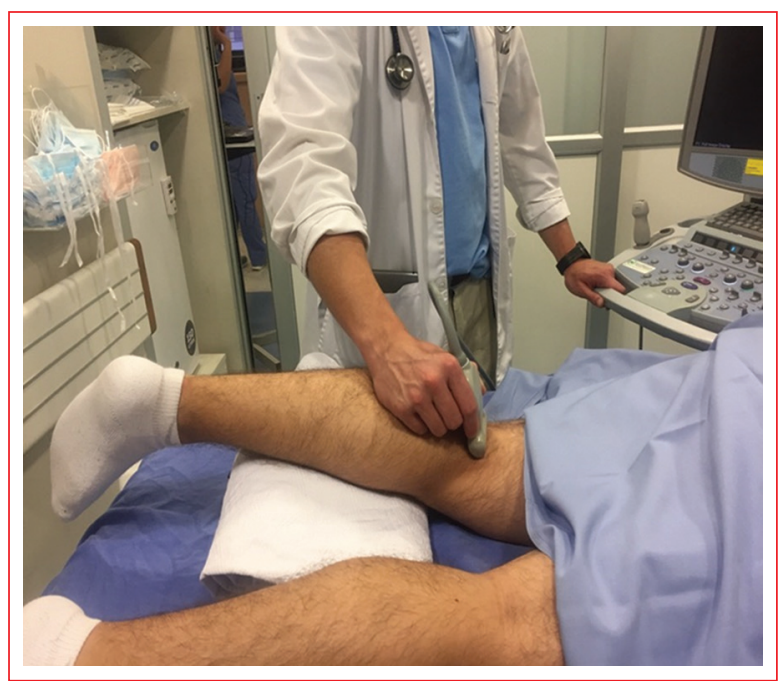

Figura 4. Exploración en fosa poplítea en prono.

El examen se llevó a cabo con la máquina de ultrasonido Mindray modelo M9 con el transductor lineal de 5-10 MHz en modalidad B. Para esto, luego de posicionar al paciente en decúbito supino se procedió a reconocer sus referencias anatómicas partiendo por el ligamento inguinal, lugar donde se comenzó la exploración de la pierna proximal. El primer punto que evaluar correspondió a la unión de la vena safena mayor con la vena femoral común (VFC) (Fig. 1), donde se realizó compresión con el transductor, con fuerza tal que deformara la vena (Figs. 2 y 3). Posterior a esto se procedió a realizar compresiones cada $1 \mathrm{~cm}$ trasladando el transductor hacia distal de forma sucesiva, identificando otro punto con alta probabilidad de formación de trombo, la bifurcación de la VFC en vena femoral superficial y profunda. Posterior a este segundo punto, se procedió a continuar realizando compresiones cada $1 \mathrm{~cm}$ hacia distal llegando hasta el canal aductor, de ser posible. Una vez evaluado el muslo, se procedió a evaluar el tercer punto, colocando el transductor en la fosa poplítea.

Para realizar la exploración a nivel poplíteo el operador posicionó al paciente según fuese lo más cómodo para la realización del examen. Esta podía ser en decúbito supino con la rodilla en flexión, decúbito lateral o prono (Fig. 4).

Una vez posicionado al paciente, en la zona más proximal a la fosa poplítea se procedió a reconocer el paquete vasculonervioso que se encuentra entre ambos cóndilos del fémur, buscando la vena poplítea (VP), lugar donde se aplicó compresión para posteriormente deslizar el transductor en sentido distal hasta la trifurcación de la VP en las tres venas de la pantorrilla.

El ultrasonido realizado por los MU se consideró positivo para TVP proximal si cualquiera de las venas analizadas no era compresible o si en estas se visualizaba un trombo endoluminal.

Si finalizado el examen no se logró la visualización óptima de algún vaso, el examen se marcó como indeterminado. Luego se procedió al registro en un documento con los datos de nombre y ficha clínica, el que posteriormente fue depositado en un buzón con acceso exclusivo a los investigadores.

Todas las exploraciones por parte del MU fueron seguidas por el estudio formal realizado por médicos radiólogos o residentes del programa de radiología, pero validados por los especialistas, lo que se considera el estándar de oro; estos abordaron la pierna completa incluyendo la identificación de TVP proximal y distal utilizando tanto la técnica de compresión como augmentación y cambios respirofásicos. Cabe destacar que el MU que realizó el examen no tuvo acceso a estos resultados.

\section{Objetivos}

El objetivo primario fue evaluar si el método educativo propuesto es útil para que MU con nula experiencia previa en USC puedan identificar la presencia o ausencia de TVP proximal comparado con la técnica descrita como estándar de oro.

\section{Registro y análisis de datos}

Los datos fueron rescatados del buzón que se encontraba en el servicio d urgencias del centro de 


\section{Médicos Emergencia Operadores de USC}

25

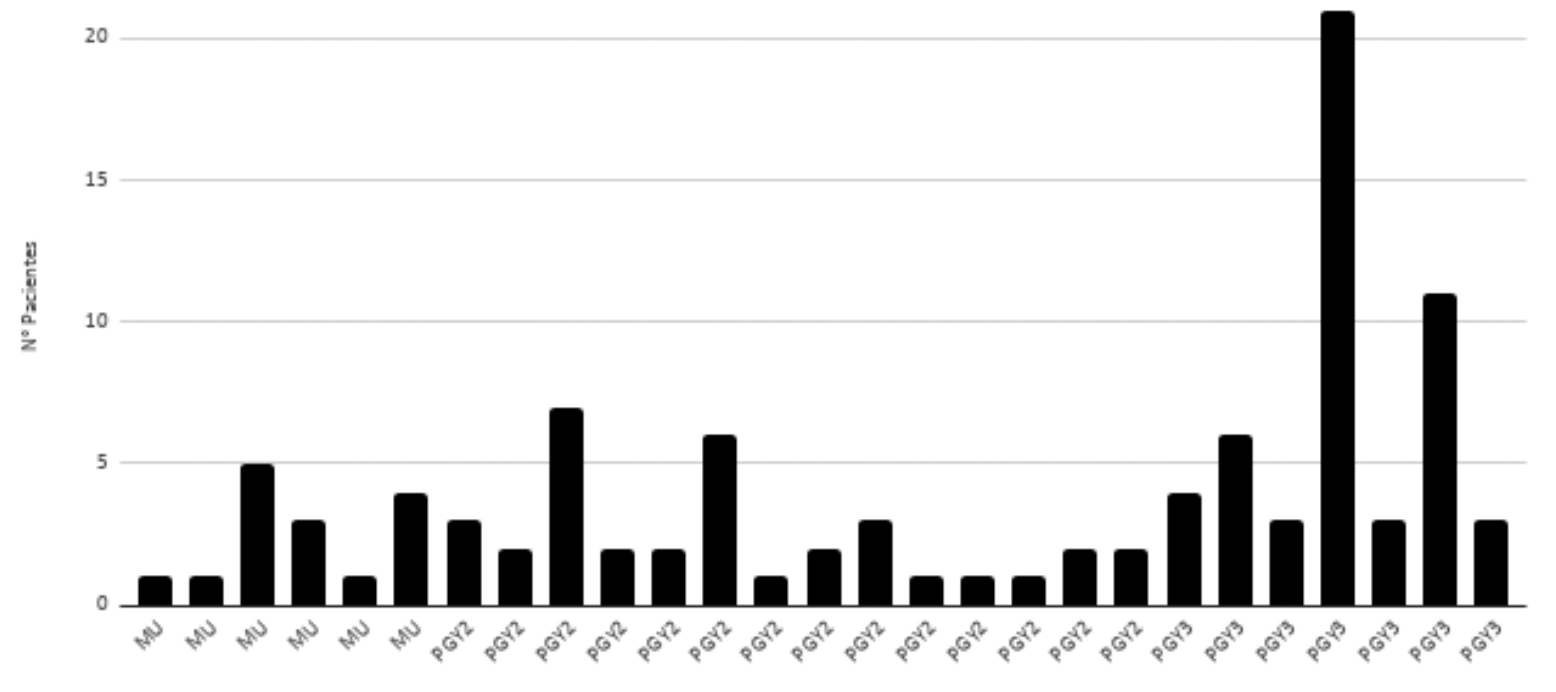

Figura 5. Pacientes enrolados por MU participante.

MU: médicos de urgencia; PGY2: residentes de medicina de urgencia segundo año; PGY3: residentes de medicina de urgencia tercer año.

estudio y registrado por parte de los investigadores en el programa Microsoft Excel (Versión 1901, Microsoft Office). Los datos fueron analizados utilizando el programa Stata versión 14.

\section{Resultados}

Se logró capacitar a 27 médicos sin experiencia previa en USC, los cuales lograron enrolar 101 pacientes entre septiembre del 2016 hasta junio del 2018. De los 101 exámenes realizados por MU, tres tuvieron resultado como indeterminado que, para efectos de este estudio, se decidió asumir como exámenes positivos, con el fin de que en el peor de los escenarios la falla en la determinación sea a favor del paciente. A todos los pacientes enrolados se les comparó contra la definición de estándar de oro.

La mediana de la edad correspondió a 59.7 años; el $58.42 \%(n=59)$ fueron mujeres (Tabla 1$)$.

Se comprobó que el $17.82 \%(n=18)$ de ellos cursaron con una TVP proximal y el 6.93\% $(n=7)$ con TVP distal. Estos últimos se consideraron negativo para TVP proximal, por encontrarse fuera de la zona examinada. Los MU identificaron correctamente a los 18 pacientes que fueron diagnosticados como TVP
Tabla 1. Caracterización de la población por sexo y edad

\begin{tabular}{|l|c|c|}
\hline Edad & Mediana: 59.7 años & Promedio: 61 años \\
\hline Sexo & Cantidad & $\%$ \\
\hline Masculino & 42 & 41.58 \\
\hline Femenino & 59 & 58.42 \\
\hline & 101 & 100 \\
\hline
\end{tabular}

Tabla 2. Comparación de USC realizado por MU y residentes vs. estándar de oro

\begin{tabular}{|c|c|c|c|}
\hline & US Rx (+) & US $\mathbf{R x}(-)$ & TOTAL \\
\hline USC (+) & 18 & 7 & 25 \\
\hline USC (-) & 0 & 76 & 76 \\
\hline & 18 & 83 & 101 \\
\hline
\end{tabular}

US: ultrasonido; USC: ultrasonido clínico; Rx: medico radiólogo o residente (estándar de oro).

proximal, sin embargo, hubo cinco exámenes que se consideraron positivos debido a discrepancias con el estándar de oro (Tabla 2). 
Tabla 3. Resultados de la técnica de USC realizada por médicos y residentes de urgencia

\begin{tabular}{|l|c|c|c|c|c|}
\hline Sensibilidad & Especificidad & VPP & VPN & LR+ & LR- \\
\hline $100 \%$ & $91.6 \%$ & $72 \%$ & $100 \%$ & 11.9 & 0 \\
\hline IC $95 \%$ & IC $95 \%$ & IC $95 \%$ & IC $95 \%$ & IC $95 \%$ & IC $95 \%$ \\
\hline $81.5-100 \%$ & $83.4-96.5 \%$ & $50.6-87.9 \%$ & - & $5.84-24.1$ & - \\
\hline
\end{tabular}

USC: ultrasonido clínico; VPP: valor predictivo positivo; VPN: valor predictivo negativo; LR: like ratio; IC: intervalo de confianza.

Posterior a los resultados, se obtuvieron valores de sensibilidad del 100\% (intervalo de confianza [IC 95\%]: 81.5-100) y una especificidad del $91.6 \%$ (IC 95\%: 83.496.5) (Tabla 3).

En la figura 5 se muestran los pacientes enrolados por MU participante. La mediana de pacientes enrolados por cada participante fue de 3.7 en un rango que fue entre 1 y 21 exámenes De los MU que participaron del estudio, 14 correspondían a residentes de segundo año PGY2, ocho a residentes de tercer año PGY3 y seis a médicos especialistas.

\section{Discusión}

La ecografía por compresión es una herramienta confiable en el diagnóstico de TVP, es por esto que algunos proponen evaluar la implementación de estrategias de entrenamiento adecuadas para maximizar las habilidades de los $\mathrm{MU}^{13}$.

Hasta la fecha no existen estudios formales donde se evalúe un método de entrenamiento o el número de estudios necesarios para tener competencia en el diagnóstico de TVP proximal mediante el uso de USC. Existen estudios sobre la efectividad diagnóstica de los distintos tipos de protocolos de USC para el diagnóstico de TVP proximal, donde se mencionan brevemente la metodología de enseñanza como el tiempo de entrenamiento, siendo tan variable con un mínimo de 10 minutos y un máximo de $24 \mathrm{~h}^{14}$.

Este es el primer estudio en evaluar la efectividad diagnóstica de un método b-learning de 30 minutos teóricos online y 90 min prácticos, en MU y operadores con nula experiencia en USC, ocupando el protocolo extendido. Todos los operadores fueron ciegos a la clínica del paciente, por lo que no hubo sesgo por la probabilidad pretest.

Es importante mencionar que los MU suelen utilizar una técnica de compresión de tres puntos, que han sido validadas por algunos estudios con niveles aceptables de sensibilidad y especificidad ${ }^{15}$. Por otro lado, otras investigaciones no han logrado niveles
Tabla 4. Grado de concordancia entre médicos de urgencias y radiólogos

\begin{tabular}{|l|c|}
\hline Concordancia & Índice de Kappa \\
\hline $93.07 \%$ & 0.79 \\
\hline
\end{tabular}

aceptables de las variables mencionadas e incluso no detectando a un tercio de los pacientes con TVP ${ }^{16,17}$. Es por esto que cabe mencionar que en los cinco casos en los que el MU encontró una TVP pero no estuvo de acuerdo con los radiólogos, se asumió que eran falsos positivos sin un seguimiento adecuado con un nuevo ultrasonido en cinco días o una angiotomografía computarizada o flebografía de extremidades inferiores.

Nuestros resultados sugieren que esta capacitación mínima tiene un rendimiento aceptable en comparación con el estándar de oro. Los resultados son similares en comparación con estudios previos, con una sensibilidad y especificidad del 96.1 y $96.8 \%$ respectivamente ${ }^{14}$.

La concordancia calculada del estudio es cercana al 93\%, con un índice de Kappa de 0.79, valor cercano para obtener una muy buena concordancia (Tabla 4). Esto pudiera deberse a la variabilidad interobservador, en contexto del examen en sí, ya que el USC es dependiente del operador.

Finalmente, según los resultados arrojados, se puede descartar con un alto nivel de sensibilidad la presencia de una TVP proximal cuando se tiene un estudio negativo. Esto es particularmente útil en escenarios donde el estándar de oro no se encuentra disponible.

Punto de relevancia además es la tardanza de la realización del examen por parte del estándar de oro, llegando en algunos casos a 14 horas $^{18}$, variable que no fue medida en el estudio.

La principal limitación de este estudio radica en que no fue posible inscribir a todos los pacientes que se presentaron en el servicio de urgencias con sospecha de TVP, el reclutamiento solo fue circunscrito al 
periodo donde se encontraba físicamente un MU previamente capacitado, existiendo periodos donde no se contaba este profesional.

Un aspecto que tener en cuenta es la amplia gama de pacientes inscritos por diferentes MU y residentes, destacando que uno de los participantes inscribió el $20 \%$ del número total de pacientes, aún así, todos ellos tenían el mismo nivel de conocimiento de USC al inicio del estudio.

Por último, otra limitante del estudio fue desconocer la prevalencia de TVP proximal y distal en la población del estudio, impidiendo de esta forma un cálculo de tamaño muestral.

\section{Financiamiento}

La presente investigación no ha recibido ninguna beca específica de agencias de los sectores públicos, comercial o sin ánimo de lucro.

\section{Conflicto de intereses}

Los autores declaran no tener conflicto de intereses.

\section{Responsabilidades éticas}

Protección de personas y animales. Los autores declaran que para esta investigación no se han realizado experimentos en seres humanos ni en animales.

Confidencialidad de los datos. Los autores declaran que han seguido los protocolos de su centro de trabajo sobre la publicación de datos de pacientes.

Derecho a la privacidad y consentimiento informado. Los autores han obtenido el consentimiento informado de los pacientes y/o sujetos referidos en el articulo.

\section{Bibliografía}

1. Lewiss RE, Pearl M, Nomura JT, Baty G, Bengiamin R, Duprey K, et al CORD-AEUS: consensus document for the emergency ultrasound milestone project. Acad Emerg Med. 2013;20(7):740-5.
2. American College of Emergency Physicians. Emergency ultrasound guidelines. Ann Emerg Med. 2009;53(4):550-70.

3. Wells PS, Hirsh J, Anderson DR, Lensing AW, Foster G, Kearon C, et al. Accuracy of clinical assessment of deep-vein thrombosis. Lancet. 1995;345(8961):1326-30

4. Wells PS, Owen C, Doucette S, Fergusson D, Tran H. Does this patient have deep vein thrombosis ? JAMA. 2006;295(2):199-207.

5. Crisp JG, Lovato LM, Jang TB. Compression ultrasonography of the lower extremity with portable vascular ultrasonography can accurately detect deep venous thrombosis in the emergency department. Ann Emerg Med. 2010;56(6):601-10.

6. Magazzini S, Vanni S, Toccafondi S, Paladini B, Zanobetti M, Giannazzo G, et al. Duplex ultrasound in the emergency department for the diagnostic management of clinically suspected deep vein thrombosis. Acad Emerg Med. 2007;14(3):216-20.

7. Röhrig S, Hempel D, Stenger T, Armbruster W, Seibel A, Walcher F, Breitkreutz R. Welche Lehrmethoden werden für die Ultraschallausbildung erwartet? : "Blended learning" auf dem Prüfstand [Which learning methods are expected for ultrasound training? Blended learning on trial]. Anaesthesist. 2014 Oct;63(10):745-52.

8. Liu Q, Peng W, Zhang F, Hu R, Li Y \& Yan W. The Effectiveness of Blended Learning in Health Professions: Systematic Review and Meta-Analysis. J Med Internet Res. 2016;18(1):e2.

9. Bernard R, Eugene B, Richard S, Rana T, Philip A. A Meta-Analysis of Blended Learning and Technology Use in Higher Education: From the General to the Applied. Journal of Computing in Higher Education, v26 n1 p87-122 Apr 2014.

10. Vodovar D, Ricard JD, Zafrani L, Weiss E, Desrentes E, Roux D. Évaluation d'un enseignement multimodal intégrant du e-learning dans l'apprentissage de la réanimation et de la médecine d'urgence au cours du second cycle des études médicales [Assessment of a newly-implemented blended teaching of intensive care and emergency medicine at Paris-Diderot University]. Rev Med Interne. 2020 Jun;41(6):368-374. French.

11. Gatica F, Rosales A. E-learning en la educación médica. Rev. Fac. Med. (Méx.) [revista en la Internet]. $2012 \mathrm{Abr}$; 55( 2 ): 27-37.

12. Needleman L, Cronan JJ, Lilly MP, Merli GJ, Adhikari S, Hertzberg BS et al. Ultrasound for Lower Extremity Deep Venous Thrombosis: Multidisciplinary Recommendations From the Society of Radiologists in Ultrasound Consensus Conference. Circulation. 2018; 137 (14): 1505-15.

13. Mumoli N, Vitale J, Giorgi-Pierfranceschi M, Sabatini S, Tulino R, Cei M, Bucherini E, Bova C, Mastroiacovo D, Camaiti A, Palmiero G, Puccetti L, Dentali F; PRACTICUS Study Investigators. General Practitioner-Performed Compression Ultrasonography for Diagnosis of Deep Vein Thrombosis of the Leg: A Multicenter, Prospective Cohort Study. Ann Fam Med. 2017 Nov; 15(6):535-539.

14. Pomero F, Dentali F, Borretta V, Bonzini M, Melchio R, Douketis JD, et al. Accuracy of emergency physician-performed ultrasonography in the diagnosis of deep-vein thrombosis: a systematic review and meta-analysis. Thromb Haemost. 2013; 109 (1): 137-45.

15. Pedraza García J, Valle Alonso J, Ceballos García P, Rico Rodríguez F, Aguayo López MÁ, Muñoz-Villanueva MDC. Comparison of the Accuracy of Emergency Department-Performed Point-of-Care-Ultrasound (POCUS) in the Diagnosis of Lower-Extremity Deep Vein Thrombosis. J Emerg Med. 2018 May;54(5):656-664.

16. Kim DJ, Byyny RL, Rice CA, Faragher JP, Nordenholz KE, Haukoos JS, Liao MM, Kendall JL. Test Characteristics of Emergency Physician-Performed Limited Compression Ultrasound for Lower-Extremity Deep Vein Thrombosis. J Emerg Med. 2016 Dec;51(6):684-690.

17. Zitek T, Baydoun J, Yepez S, Forred W, Slattery DE. Mistakes and Pitfalls Associated with Two-Point Compression Ultrasound for Deep Vein Thrombosis. West J Emerg Med. 2016 Mar;17(2):201-8.

18. Kory PD, Pellecchia CM, Shiloh AL, Mayo PH, DiBello C, Koenig S. Accuracy of ultrasonography performed by critical care physicians for the diagnosis of DVT. Chest. 2011 Mar;139(3):538-542. 